\title{
Arabinogalactan Glycosyltransferases: Enzyme Assay, Protein-Protein Interaction, Subcellular Localization, and Perspectives for Application
}

\author{
Naomi Geshi \\ Department of Plant and Environmental Sciences, Faculty of Science, University of Copenhagen, Thorvaldsensvej 40, \\ 1871 Frederiksberg C, Denmark
}

Correspondence should be addressed to Naomi Geshi; ngeshi@outlook.com

Received 8 May 2014; Revised 17 August 2014; Accepted 21 August 2014; Published 10 September 2014

Academic Editor: Scott Finlayson

Copyright (C) 2014 Naomi Geshi. This is an open access article distributed under the Creative Commons Attribution License, which permits unrestricted use, distribution, and reproduction in any medium, provided the original work is properly cited.

\begin{abstract}
Arabinogalactan proteins (AGPs) are abundant extracellular proteoglycans that are found in most plant species and involved in many cellular processes, such as cell proliferation and survival, pattern formation, and growth, and in plant microbe interaction. AGPs are synthesized by posttranslational $O$-glycosylation of proteins and attached glycan part often constitutes greater than $90 \%$ of the molecule. Subtle altered glycan structures during development have been considered to function as developmental markers on the cell surface, but little is known concerning the molecular mechanisms. My group has been working on glycosylation enzymes (glycosyltransferases) of AGPs to investigate glycan function of the molecule. This review summarizes the recent findings from my group as for AtGalT31A, AtGlcAT14A-C, and AtGalT29A of Arabidopsis thaliana with a specific focus on the (i) biochemical enzyme activities; (ii) subcellular compartments targeted by the glycosyltransferases; and (iii) protein-protein interactions. I also discuss application aspect of glycosyltransferase in improving AGP-based product used in industry, for example, gum arabic.
\end{abstract}

\section{Introduction}

Arabinogalactan proteins (AGPs, AG proteins) are plantspecific proteoglycans that are found in most of the analyzed plant species. The main component of AGPs is AG glycan, which often constitutes greater than $90 \%$ of the molecule. Studies using monoclonal antibodies recognizing different epitopes of AG glycans have demonstrated a temporal and a spatial appearance of certain subpopulations of AG glycans during plant development (for review, see [1]). In animal cells, the structural diversities of cell-surface proteoglycans underlie their various functions, for example, interaction with hormone, extracellular matrix components, and enzymes, and play an important role in signal transduction and/or cell-cell communication and adhesion (for review, see [2]). Analogous mode of action is expected for a specific AG subpopulation during plant development, but little is known regarding the molecular mechanisms. My group has been working on the biosynthetic enzymes of AG glycans to understand the developmentally regulated biosynthesis of AG glycans.
AG glycans are synthesized on core proteins by posttranslational modifications (O-glycosylation). The structure of AG glycans is complex and heterogeneous, changes throughout development, and commonly consists of $\beta$-1,3-linked galactans as main chains that are substituted with various lengths of $\beta$-1,6-galactan side chains, which are further decorated by arabinose and other sugars, such as (methyl) glucuronic acid (GlcA), rhamnose, and fucose. Glycosylation is catalyzed by large number of glycosyltransferases (GTs) in the secretory pathway.

To date, two $\alpha$-1,2-fucosyltransferases (AtFUT4 and AtFUT6) [3], three galactosyltransferases (AtGALT2, AtGALT31A, and AtGALT29A) [4-6], and three glucuronosyltransferases (AtGlcAT14A, B, and C) [7, 8], all from $A r$ abidopsis thaliana, have been characterized (for review, see [9]). The mutation in the RAY1 gene leads to a reduction in the arabinose residues in AGPs [10] and, as expected, encodes the activity of arabinosyltransferase. However, the recombinant protein made $\beta$-linked arabinose instead of $\alpha$-linked arabinose that is present in AGPs; therefore, the 
(1) Identification of donor-substrate

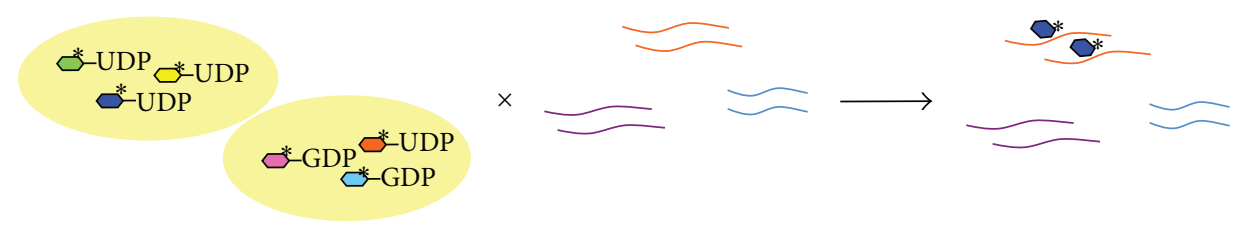

(2) Identification of acceptor-substrate

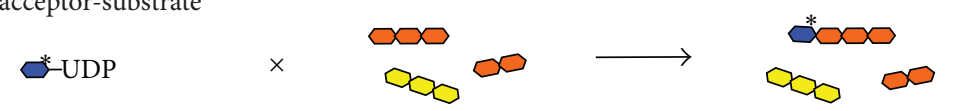

FIGURE 1: Screening of glycosyltransferase activities. Recombinant protein is tested for glycosyltransferase activity in the presence of broad range of donor-substrates and acceptor-mixture (1). Acceptor molecule is identified by analyzing the product made on the acceptor-mixture in (1) by testing susceptibility to structure-specific hydrolases and/or testing structure-defined oligosaccharides for the glycosyltransferase activity (2).

involvement of this enzyme in the arabinosylation of AGPs seems not as direct as $\alpha$-arabinosyltransferase.

Among those GTs that are listed above, we have characterized AtGalT31A, AtGalT29A, AtGlcAT14A, AtGlcAT14B, and AtGlcAT14C. We took a cross-disciplinary approach to characterize the biochemical properties of the enzymes and their cell biological aspects and used basic research to advance towards a more biotechnological application of the enzymes. This review summarizes the main research outcomes from my group and discusses future perspectives in this research field.

\section{Main Text}

2.1. Identification and Biochemical Characterization of Arabinogalactan Glycosyltransferases from Arabidopsis thaliana. GTs are difficult enzymes to characterize because (i) these enzymes are generally expressed at low levels, (ii) redundant activities mask the mutant phenotype, and (iii) pleiotropic effects confuse the relationship between the mutant phenotype and the enzyme activity that is encoded by the mutated gene. It reflects the fact that enzyme activities that are encoded by approximately 20 genes have biochemically been characterized in Arabidopsis thaliana, among more than 450 putative GTs that have been deposited into the carbohydrate active enzyme database (CAZy, http://www.cazy.org/).

We have used a forward genetics approach for putative GTs from Arabidopsis thaliana, taking advantage of the completed genome sequencing in 2001 and availability of a large amount of -omics data for this species. Because $A G$ is a unique structure in plants and it is difficult to predict GT candidates from the primary sequences, we developed a screening method for GTs that are related to AG biosynthesis (illustrated in Figure 1). First, we prepared a heterogeneous mixture of various AG oligosaccharides from microsomes after expressing AGP synthetic peptides in tobacco leaves. Using the AG acceptor-mixture, we measured the GT activity from wide range of ${ }^{14}[\mathrm{C}]$-labeled donor-substrates. Next, we attempted to identify acceptor molecules by analyzing susceptibility of the resulted ${ }^{14}[\mathrm{C}]$-sugar-incorporated products by structure-specific hydrolases or by testing the structuredefined oligosaccharides as acceptors. There are several available AG-specific hydrolases, for example, endo- $\beta$-1,6-galactanase, exo- $\beta$-1,3-galactanase, $\alpha$-arabinofuranosidase, $\beta$-glucuronidase, and $\alpha$-rhamnosidase, which are extremely useful when analyzing the AG products (for review, see [9]).

Using this method, we identified AtGalT31A, AtGalT29A, and AtGlcAT14A, 14B, and 14C as AG GTs.

AtGALT31A, which is encoded by At1g32930, was identified as a galactosyltransferase that is involved in elongating the $\beta$-1,6-galactan of the AG side chains [5]. The soluble catalytic domain expressed in $E$. coli and the full-length protein expressed in $N$. benthamiana demonstrated a transfer of ${ }^{14}[\mathrm{C}]-\mathrm{Gal}$ from UDP- ${ }^{14}[\mathrm{C}]-\mathrm{Gal}$ to the heterologous mixture of AG acceptors. The ${ }^{14}[\mathrm{C}]$-incorporated products were susceptible to the treatment of endo- $\beta$-1,6-galactanase and resulted in ${ }^{14}[\mathrm{C}]$-galactose and ${ }^{14}[\mathrm{C}]$-galactobiose but were not susceptible to the exo- $\beta$-1,3-galactanase treatment. These results indicate that the transferred ${ }^{14}[\mathrm{C}]-\mathrm{Gal}$ was a part of $\beta$-1,6-galactan, which is longer than galactotriose, and that this enzyme acts to elongate $\beta$-1,6-galactan but not $\beta$-1,3-galactan. The mutation of this gene resulted in an embryo-lethal phenotype (see below), and we could not confirm the in vivo effect of the AG structure by knocking out this gene. We named the enzyme AtGalT31A (Arabidopsis thaliana galactosyltransferase from family 31).

AtGlcAT14A (At5g39990) and AtGalT29A (At1g08280) were coexpressed with AtGalT31A (At1g32930) (GeneCAT [12]), and we selected as candidate GTs that are involved in the biosynthesis of AG. We investigated the encoding activities of these GTs by heterologous expression and in vitro enzyme assays as were performed in the study of AtGalT31A.

The soluble catalytic domain of AtGlcAT14A (At5g39990) expressed in Pichia pastoris demonstrated a transfer of ${ }^{14}[\mathrm{C}]$ GlcA from UDP- ${ }^{14}[\mathrm{C}]-$ GlcA to both $\beta-1,3-$ and $\beta$-1,6-linked galactooligosaccharides [7]. A mutant analysis revealed a reduction of the corresponding structure in isolated AG (see below), indicating that this is enzyme responsible for the addition of GlcA to the terminal $\beta$-1,6-galactan side chains and of a single GlcA to the $\beta$-1,3-galactan main chain of AG. 
We named the enzyme AtGlcAT14A (Arabidopsis thaliana glucuronic acid transferase from family 14). The CAZy family GT14 contains 11 proteins, including AtGlcAT14A. When we analyzed the recombinant proteins for all of the 11 isoforms, At5g15050 and At2g37585 also demonstrated glucuronosyltransferase activity by adding GlcA to $\beta-1,3$ - and $\beta$-1,6-linked galactans [8]. Therefore, we named At5g15050 and At2g37585 as AtGlcAT14B and AtGlcAT14C, respectively.

For AtGalT29A (Atlg08280), we expressed the full-length protein in Nicotiana benthamiana and demonstrated a transfer of ${ }^{14}[\mathrm{C}]-\mathrm{Gal}$ from UDP- ${ }^{14}[\mathrm{C}]-\mathrm{Gal}$ to a mixture of AG acceptors [6]. The products were susceptible to endo- $\beta-1,6-$ galactanase, and the release of small oligosaccharides was promoted by cotreatment with $\alpha$-arabinofuranosidases. This result indicates that the incorporated ${ }^{14}[\mathrm{C}]$-Gal was a part of $\beta$-1-6-galactooligosaccharides that were longer than galactotriose with and without the substitution of arabinose. The ${ }^{14}[\mathrm{C}]$-incorporated product was also susceptible to exo- $\beta-1,3-$ galactanase, and the treatment released ${ }^{14}[\mathrm{C}]$-disaccharide but not ${ }^{14}[\mathrm{C}]$-monosaccharide, indicating that the enzyme activity on $\beta$-1,3-galactan is a $6{ }^{14}[\mathrm{C}]$-Gal branch-forming activity rather than a $\beta-1,3$-galactan elongating activity. Thus, AtGALT29A possesses $\beta$-1,6-GalT activity elongating $\beta$-1,6galactan and making branches on $\beta$-1,3-galactan, and we named the enzyme AtGalT29A (Arabidopsis thaliana galactosyltransferase from family 29).

We found that AtGALT29A forms a protein complex with AtGALT31A when expressed ectopically in $N$. benthamiana and that the enzyme complex exhibited an increased level of $\beta-1,6-$ GalT activity as described above compared to the single AtGALT29A (the details are described below). Additionally, a proteomics analysis identified phosphorylated Y144, and a site-directed mutagenized construct on Y144 affected the subcellular targeting and enzyme activity of AtGALT29A (the details are described below).

2.2. Mutant Analysis. A T-DNA insertional mutant of At1g32930 (AtGalT31A) was embryo-lethal [5]. In the embryo proper, the AtGALT31A mRNA was specifically located in the suspensor, while abnormal cell division was observed in the hypophysis during the globular stage of the embryo in the atgalt31a mutant lines. The atgalt31a mutants arrested further development of the embryo proper, which resulted in the embryo-lethal phenotype. Thus, functional AtGALT31A is essential for normal embryo development. However, how the spatially distant expression of AtGALT31A in the suspensor influences the cell division in the hypophysis remains unknown.

A T-DNA insertional mutant of At5g39990 (AtGlcAT14A) exhibited an increased elongation rate during seedling growth under both light and dark conditions [7]. In the isolated AG from the atglcat14a lines, the level of GlcA substitution on both the $\beta$-1,6-galactan side chains and $\beta-1,3-$ galactan main chains was reduced. Moreover, we observed an increase in the 3-, 6-, and 3,6-linked Gal and a decrease in the 2-, 3-, 5-, and 2,5-linked Ara in the mutant AG. We interpreted the increase in the 6- and 3,6-Gal linkages as a result of the increased availability of $O 6$ in both $\beta-1,3-$ linked and $\beta$-1,6-linked Gal for elongation of galactans due to the reduction in the activity of AtGlcAT14A, since both enzymes act on the same acceptor site of Gal. An apparent reduction of Ara might be seen if the amount of Ara was unchanged while elongating $\beta$-1,6-galactan when the sugar levels were calculated as mol\% relative to the total amount, but this hypothesis remains to be elucidated.

An analysis of knock-out mutants for At1g08280 (AtGALT29A), At5g15050 (AtGlcAT14B), and At2g37585 (AtGlcAT14C) is underway. It has been suggested that the GlcA residues on the AG side chains bind to extracellular calcium and play roles as Ca capacitors [13] and that AG GlcAT activities may be important to guarantee GlcA residues in the AGPs. Calcium plays a central role in cell elongation and growth in plants. We did not observe a detectable growth phenotype in atglcat14a in addition to the enhanced cell elongation in the seedlings [7], but obviously AtGlcAT14B and C likely have redundant activities with AtGlcAT14A in plants [8]. Therefore, it is necessary to investigate double and triple mutants to understand the role of the GlcA residues in AG glycans.

2.3. Protein-Protein Interactions between AtGALT29A and AtGALT31A Result in Increasing $\beta-1,6$-Galactosyltransferase Activities. In mammalian cells, it is well known that enzymes that are involved in protein glycosylation interact with each other and form "assembly lines" in the secretory pathway [14]. We, therefore, investigated the protein-protein interactions among AtGALT31A, AtGALT29A, and AtGLCAT14A that were ectopically expressed as C-terminally fluorescentlabeled constructs in N. benthamiana by analyzing the Förster resonance energy transfer (FRET) between the fluorescent donor and acceptor proteins [6]. We established the acceptor photobleaching FRET method to study the protein-protein interactions between GTs tagged with mCer3 (donor fluorophore, monomeric cyan fluorescent protein 3) and YFP (acceptor fluorophore, yellow fluorescent protein) that reside in the Golgi apparatus by stop cytoplasmic streaming by an inhibitor for actin polymerization, Cytochalasin D (CytD) [15]. The pixel-by-pixel FRET efficiencies were calculated by [16] using the ImageJ [17] plug-in. Because the 0 scale is set for the FRET efficiency obtained from the donor-only control, any values greater than 0 indicate a close proximity of less than $10 \mathrm{~nm}$ and, thus, the presence of a protein-protein interaction [16].

Using this method, we demonstrated interactions between AtGALT29A-mCer and AtGALT29-YFP (homodimer), AtGALT31A-mCer and AtGALT31A-YFP (homodimer), and AtGALT29A-mCer (or -YFP) and AtGALT31A-YFP (or -mCer) (heterodimer) [6]. However, there were no interactions between a combination of AtGALT31A-mCer3 and AtGlcAT14A-YFP [7]. The FRET efficiency between AtGAL29A and AtGLCAT14A was greater than 0 (13\%, [6]), but these two proteins often do not colocalize, suggesting that this interaction is likely less important than the interactions that were found between AtGALT29A and AtGALT31A, in which two proteins colocalize at a high 
frequency. Furthermore, based on the level of the FRET efficiencies, it has been suggested that (i) AtGALT31A is less capable of forming dimers than is AtGALT29A and (ii) the heterodimer between AtGALT29A and AtGALT31A is the preferred form when both AtGALT29A and AtGALT31A are available, and we anticipate the preferred forms of GTs in the following order: AtGALT29A/AtGALT31A heterodimer, AtGALT29A homodimer, AtGALT31A homodimer, and AtGALT31A monomer [6].

We could purify the protein complex containing both AtGALT29A and AtGALT31A by coimmunoprecipitation using an epitope tag of one of the recombinant proteins [6]. The singly expressed recombinant protein of AtGALT31A and AtGALT29A possess $\beta-1,6-$ GalT activity elongating $\beta$ 1,6-galactan (AtGALT29A also possesses $\beta-1,6-$ GalT activity adding single 6 -Gal to $\beta$-1,3-galactan) at low levels, but the protein complex containing AtGALT29A and AtGALT31A demonstrated an increased level of $\beta-1,6-$ GalT activities both elongating $\beta$-1,6-galactan and adding single 6 -Gal to $\beta$ 1,3-galactan compared to the AtGALT29A or AtGALT31A single enzyme. According to the FRET data, it is conceivable that AtGALT29A and AtGALT31A are present as a heterodimer in the coimmunoprecipitated sample, while they are present as homodimers when they are expressed alone. The results indicated a cooperative action of AtGALT29A and AtGALT31A by forming a heterodimer. It is conceivable that the protein-protein interaction can more rapidly adapt to the environment compared to the transcriptional controls. $\beta-1,6-$ Galactan is detected specifically in the tips of the root hair by a specific antibody [18]; therefore, the interaction between AtGALT29A and AtGALT31A may contribute to the specific appearance of the $\beta$-1,6-galactan side chains of $A G$ in such a limited portion of tissues during plant development. The investigation of other GTs that are involved in the AG biosynthetic pathway should address the importance of the role of protein-protein interactions in the biosynthesis of AG glycans.

2.4. Arabinogalactan Glycosyltransferases Target Uncharacterized Subcellular Compartments Which May Be a Part of an Unconventional Protein Secretion Pathway. When we investigated the subcellular localization of AG GTs by the ectopic expression of C-terminal fluorescence-fusion proteins in $N$. benthamiana, we found that AG GTs are localized not only to the Golgi apparatus $(\sim 1.0 \mu \mathrm{m}$ diameter $)$ but also to yet uncharacterized smaller compartments $(\sim 0.5 \mu \mathrm{m}$ diameter $)$ [11] (Figure 2). All three of the studied AG GTs (AtGALT31A, AtGALT29A, and AtGlcAT14A) showed dual localization in the Golgi and these small compartments, but AtGALT31A was frequently localized to the small compartments $(\sim 80 \%$ of the total AtGALT31A population when expressed in $N$. benthamiana). However, the $N$-glycosylation enzymes ( $N$ acetylglucosaminyltransferase I, GnTI; $\alpha$-mannosidase II, GMII; and sialyltransferase, ST) were almost all localized to the Golgi and were rarely found in the small compartments where AtGALT31A was localized. Therefore, the small compartments appeared to be rather dedicated to a portion of AG biosynthesis but not to $N$-glycan processing. This

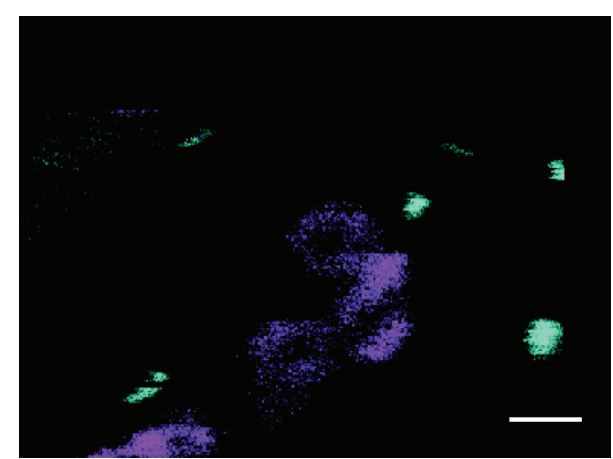

Figure 2: The AtGALT31A-mCer3-localized small compartments (cyan) and the mannosidase II-mRFP-localized Golgi cisternae (magenta) when transiently expressed in $N$. benthamiana leaves. Scale bar $=1 \mu \mathrm{m}$.

organization is different from that of mammalian cells, in which both the $\mathrm{N}$ - and $\mathrm{O}$-glycosylation enzymes are located in the Golgi but are separated by forming protein complexes within the pathway but not across the pathways [19]. Considering the presence of more than twice of glycosyltransferases in plants (e.g., more than 450 from Arabidopsis thaliana in CAZy database) than in mammals (e.g., approximately 200 from Homo sapiens in CAZy database), plant cells most likely have evolved multiple management systems to organize the many different glycosylation processes, which not only form protein complexes within the pathway (protein-protein interactions across the pathways have not been studied in plants) but also localize the pathway in a spatially different organelle.

The AtGALT31A-localized small compartments are of a similar size as the trans-Golgi network (TGN) defined by SYP61, but AtGALT31A did not colocalize with SYP61 [11]. Additionally, the AtGALT31A-localized small compartments did not colocalize with the FM4-64-stained early endosome [11]. Instead, AtGALT31A partially colocalized with EXO70E2, a newly identified marker for an exocyst-positive organelle (EXPO) that is present in Arabidopsis and tobacco cells [20]. The EXPO represents an unconventional secretory pathway (UPS) in plants, apart from the conventional secretory pathway from the ER, the Golgi apparatus, and the TGN to the plasma membrane (for review, see [21]).

The AtGALT31A-localized small compartments were rather insensitive to the treatments of Brefeldin $\mathrm{A}$ and Wortmannin [11], two well-known inhibitors of different parts of vesicle trafficking in the secretory pathway. This feature has also been observed in EXPO as evidence of being a Golgi-independent pathway [20]. The overall results implicate the AtGALT31A-localized small compartments as distinct from the Golgi, SYP61-localized TGN, FM4-64stained early endosomes, and Wortmannin-vacuolated prevacuolar compartments but as most likely a part of UPS pathway together with EXPO in plants.

2.5. The Replacement of Tyrosine 144 by Other Amino Acids and Coexpression with AtGALT31A Effects on Subcellular Targeting of AtGALT29A. Proteomics studies identified the 
TABLE 1: AtGALT29A with or without the mutagenesis of Y144. ${ }^{1}$ Interaction with AtGALT31A as analyzed by FRET acceptor photobleaching $[6,11]$; the numbers in brackets indicate the FRET efficiencies; ${ }^{2}$ frequency (\%) of the subcellular targeting of the small compartments of $\sim 0.5 \mu \mathrm{m}$ in diameter when the construct was expressed solely or coexpressed with AtGALT31A [11]; the numbers in brackets indicate the colocalization frequency with AtGALT31A within the small compartments.

\begin{tabular}{lcrr}
\hline & \multicolumn{2}{c}{ Interaction with AtGALT31A $^{1}$} & \multicolumn{2}{c}{ Localization to small compartments } \\
& & Expressed solely & Coexpressed with AtGALT31A \\
\hline AtGALT29A & Yes (18\%) & $25 \%$ & $39 \%(38 \%)$ \\
Y144A & Yes (9\%) & $60 \%$ & $79 \%(66 \%)$ \\
Y144E & Yes (10\%) & $70 \%$ & $66 \%(64 \%)$ \\
\hline
\end{tabular}

phosphorylated Tyr at 144 (Y144) on a peptide that was derived from AtGALT29A [22], and we investigated the significance of Y144 by replacing Y144 with A or E (Y144A and Y144E, resp.) by site-directed mutagenesis. AtGALT29A possesses approximately 10 possible phosphorylation sites in its sequence; however, the Y144 is the only site where the phosphorylation has been detected. The predicted 3D structure indicates that the Y144 site is located in the peripheral surface of the globular catalytic domain of AtGALT29A. Other GTs, for instance, AtGALT31A, do not possess a Tyr in the corresponding position.

Subcellular targeting was affected by the Y144A and Y144E mutagenesis of recombinant AtGALT29A. AtGALT29A without mutagenesis localizes approximately $25 \%$ of the whole population to the small compartments of $\sim 0.5 \mu \mathrm{m}$ diameter, while the targeting frequency increased to $60 \%$ and $70 \%$ in the $\mathrm{Y} 144 \mathrm{~A}$ and $\mathrm{Y} 144 \mathrm{E}$ constructs, respectively, when expressed transiently in $N$. benthamiana [11] (Table 1). Because both mutageneses showed an increased frequency of targeting to the small compartments, Y144 itself appeared to play a role in determining the subcellular targeting rather than the electrostatic status of Y144.

The subcellular distribution of AtGALT29A also changed when the construct was expressed solely or coexpressed with the interaction partner AtGALT31A. The results that are described above are from the sole expression of the respective construct in $N$. benthamiana. When these constructs were coexpressed with AtGALT31A, targeting to the small compartments increased from $25 \%$ to $40 \%$ in AtGALT29A and from $60 \%$ to $79 \%$ in AtGALT29A Y144A, while the distribution of Y144E in the small compartments was roughly the same or slightly decreased to $66 \%$ compared to when expressed alone (70\%) [11] (Table 1). We suspected that the increased targeting to the small compartments was due to the increased interaction with AtGALT31A; however, the FRET efficiencies between AtGALT31A-mCer3 and AtGALT29A Y144A-YFP or Y144E-YFP were lower (9\% and 10\%, resp., [11]) than the value between AtGALT31A-mCer3 and AtGALT29A-YFP (18\%) [6] (Table 1). Therefore, Y144A and Y144E appeared less capable of forming a heterodimer with AtGALT31A than the unmodified AtGALT29A, and it is unlikely that the increased targeting of Y144A and Y144E to the small compartments was led by an increased interaction with AtGALT31A. Currently, we do not know the factors controlling the subcellular targeting of AtGALT29A Y144A or Y144E.
Taken together, Y144 appears to be important in determining the subcellular distribution of AtGALT29A and when both AtGALT29A and AtGALT31A are present, the two proteins most likely interact and increase their localization to the small compartments. When AtGALT29A forms a complex with AtGALT31A, the protein complex exhibits an increased level of GalT activity compared to that of the AtGALT29A single enzyme [6]. We have preliminary data demonstrating an increase in the GalT enzyme activity in the recombinant Y144A protein and a decreased activity in the recombinant Y144E protein compared to the level of AtGALT29A activity without mutagenesis. Therefore, it is interesting to investigate the relationships between (i) the charge status of Y144 and (ii) the heterodimer formation with AtGALT31A and whether these factors work additively, synergistically, or antagonistically in controlling the GalT activity of AtGALT29A.

2.6. Gum Arabic Harboring an Increased Amount of GlcA by the In Vitro Action of AtGlcAT14A Improved the Emulsifier Property. Gum arabic is the exudate of Acacia senegal and Acacia seyal produced upon wounding and is used widely as a food additive (E414 in Europe, GRAS in FDA in USA). Because gum arabic does not form a viscous solution up to quite high concentrations (Newtonian flow up to $40 \%(\mathrm{w} / \mathrm{v}))$, this product is particularly superior as an oil-inwater emulsifier in the beverage area. Gum arabic is a mixture of heterogeneous substances, of which AGP is essential for its function as an emulsifier by having both hydrophobic protein and hydrophilic carbohydrate portions that interact with oil droplets and aqueous solutions, respectively [23]. The carbohydrate portions of gum arabic are believed to provide a strong steric barrier against the flocculation and coalescence of oil-in-water emulsions [24, 25].

Because we found that the commercial gum arabic (Sigma-Aldrich) serves as a good acceptor for the reaction of AtGlcAT14A (GlcA was incorporated from UDP-GlcA up to $1 / 3$ of the total gum arabic weight), we characterized the oilin-water emulsions of the modified gum arabic by the action of AtGlcAT14A and compared these emulsions to unmodified gum arabic. We found that the oil-in-water emulsions that were made by the enzyme-modified gum arabic were slightly smaller in size and remarkably more stable compared to the emulsions that were made by the unmodified gum arabic [26]. This result is likely due to the additionally incorporated 
GlcA by the action of AtGlcAT14A providing extra charges to the carbohydrate surface of the oil-in-water emulsions that are mediated by gum arabic, likely resulting in an increase of the electrical repulsion of each emulsion and preventing interactions between each other, leading to flocculation and coalescence. Thus, this modification appears promising for improving the emulsifier property of gum arabic.

2.7. Conclusions and Future Perspectives. We have characterized the arabinogalactan glycosyltransferases AtGALT31A, AtGLCAT14A, and AtGALT29A (later also AtGlcAT14B and C) for their biochemical enzyme activities and cell biological aspects of the proteins (protein-protein interactions, subcellular targeting, etc.) and have provided novel insight into our understanding of AGP biosynthesis. Heterodimer formation between AtGALT31A and AtGALT29A may be a rapid way to respond to a requirement of the synthesis of $\beta$-1,6-galactan and might be a regulatory mechanism to serve subtly changed AG glycans during plant development. The importance of such systems working in other GTs in AGP glycosylation remains to be elucidated. Furthermore, we identified small subcellular compartments that are uniquely targeted by AG GTs (protein $\mathrm{O}$-glycosylation) but not by protein $\mathrm{N}$ glycosylation enzymes. This result suggests a different level of organization of $O$ - and $N$-glycosylation enzymes in plants compared to that in mammalian cells, most likely a result of the adaptation to a large number of glycosylation processes in plants, for example, by the presence of cell walls. Moreover, we found an overlap with the EXPO and the small compartments based on a colocalization study and suggested a possibility of the small compartments being involved in an unconventional protein secretion pathway. EXPO is uniquely composed of double membranes and releases inner single-membranebound vesicles into the extracellular space after the outer membrane fuses to the plasma membrane [20]. EXPO may serves an attractive alternative transport system and glycosylation localization in addition to the conventional secretory pathway through the Golgi apparatus, which has been used to produce recombinant glycoconjugates by now. It is well known that the subcellular localization of the glycosylation is necessary to investigate the detailed domain architectures of the proteins that reside in the small compartments and EXPO for future applied research, for example, to produce a foreign (glycosylated) peptide to be excreted outside of the cells. If we have knowledge of the domains defining the subcellular targeting and the domains that are involved in protein-protein interactions, we could manipulate the glycan structure by domain swapping (successful examples described in a review in [27]). A suspension cell culture system is worth investigating as a candidate host because these cells naturally produce AGPs in a very large amount and secrete AGPs to the medium [28]; therefore, these cells should serve a good base for further genetic engineering.

GTs are most likely not the traditional industrial enzymes for use in in vitro reactions because they require an expensive donor-substrate (NDP-sugar). However, an in vivo approach (metabolic engineering) may be a promising approach to modify the glycan structure of useful glycoconjugates. We have shown that the additional GlcA incorporation to gum arabic by the in vitro incubation of recombinant AtGlcAT14A in the presence of UDP-GlcA improves the emulsifier property of gum arabic. The in planta modification of gum arabic by the co- or overexpression of AtGlcAT14A would be a useful approach to produce enzyme-modified gum arabic. The application of genetics in acacia trees may not be an easy solution, but Professor Kieliszewski's group at Ohio University succeeded in producing a synthetic gum arabic variant by expressing a synthetic peptide based on the sequencemotif that was obtained from a purified gum arabic glycoprotein in tobacco suspension BY2 cells [29-31]. The (arabino)glycosylated synthetic peptide that was produced by the action of tobacco endogenous enzymes performed equally as or even better than commercial gum arabic at emulsifying and stabilizing the activities of the oil-in-water emulsions [32]. Therefore, AtGlcAT14A could be co-(over)expressed in the BY2 lines expressing synthetic gum arabic peptide to produce a gum arabic variant harboring extra amounts of GlcA.

In summary, AGPs are interesting molecules because of their importance not only in plant physiology but also as a product, for example, gum arabic. GTs that are involved in AGP biosynthesis have only begun to be identified and characterized. Further investigations of the biosynthetic pathway, subcellular localization, significance of protein-protein interactions, and posttranslational modifications (phosphorylation) will not only strengthen our knowledge of plant physiology but also provide important tools for making use of the biosynthetic pathway for the production of useful glycoconjugates, such as pharmaceuticals.

\section{Conflict of Interests}

The author declares that there is no conflict of interests regarding the publication of this paper.

\section{References}

[1] G. J. Seifert and K. Roberts, "The biology of arabinogalactan proteins," Annual Review of Plant Biology, vol. 58, pp. 137-161, 2007.

[2] N. Perrimon and M. Bernfield, "Cellular functions of proteoglycans-an overview," Seminars in Cell and Developmental Biology, vol. 12, no. 2, pp. 65-67, 2001.

[3] Y. Wu, M. Williams, S. Bernard, A. Driouich, A. M. Showalter, and A. Faik, "Functional identification of two nonredundant Arabidopsis $\alpha(1,2)$ fucosyltransferases specific to arabinogalactan proteins," Journal of Biological Chemistry, vol. 285, no. 18, pp. 13638-13645, 2010.

[4] D. Basu, Y. Liang, X. Liu et al., "Functional identification of a hydroxyproline-O-galactosyltransferase specific for arabinogalactan protein biosynthesis in arabidopsis," The Journal of Biological Chemistry, vol. 288, no. 14, pp. 10132-10143, 2013.

[5] N. Geshi, J. N. Johansen, A. Dilokpimol et al., "A galactosyltransferase acting on arabinogalactan protein glycans is essential for embryo development in Arabidopsis," Plant Journal, vol. 76, pp. 128-137, 2013.

[6] A. Dilokpimol, C. P. Poulsen, G. Vereb, S. Kaneko, A. Schulz, and N. Geshi, "Galactosyltransferases from Arabidopsis thaliana in the biosynthesis of type II arabinogalactan: molecular 
interaction enhances enzyme activity," BMC Plant Biology, vol. 14, p. 90, 2014.

[7] E. Knoch, A. Dilokpimol, T. Tryfona et al., "A $\beta$-glucuronosyltransferase from Arabidopsis thaliana involved in biosynthesis of type II arabinogalactan has a role in cell elongation during seedling growth," The Plant Journal, vol. 76, pp. 1016-1029, 2013.

[8] A. Dilokpimol and N. Geshi, "Arabidopsis thaliana glucuronosyltransferase in family GT14," Plant Signaling \& Behavior, vol. 9, Article ID e28891, 2014.

[9] E. Knoch, A. Dilokpimol, and N. Geshi, "Arabinogalactan proteins: focus on carbohydrate active enzymes," Frontiers in Plant Science, vol. 5, article 198, 2014.

[10] S. Gille, V. Sharma, E. E. K. Baidoo, J. D. Keasling, H. V. Scheller, and M. Pauly, "Arabinosylation of a Yariv-precipitable cell wall polymer impacts plant growth as exemplified by the Arabidopsis glycosyltransferase mutant rayl," Molecular Plant, vol. 6, no. 4, pp. 1369-1372, 2013.

[11] C. P. Poulsen, A. Dilokpimol, G. Mouille, M. Burow, and N. Geshi, "Arabinogalactan glycosyltransferases target to a unique subcellular compartment that may function in unconventional secretion in plants," Traffic, 2014.

[12] M. Mutwil, J. Obro, W. G. T. Willats, and S. Persson, "GeneCAT-novel webtools that combine BLAST and co-expression analyses," Nucleic Acids Research, vol. 36, pp. W320-W326, 2008.

[13] D. T. A. Lamport and P. Várnai, "Periplasmic arabinogalactan glycoproteins act as a calcium capacitor that regulates plant growth and development," New Phytologist, vol. 197, no. 1, pp. 58-64, 2013.

[14] C. L. De Graffenried and C. R. Bertozzi, "The roles of enzyme localisation and complex formation in glycan assembly within the Golgi apparatus," Current Opinion in Cell Biology, vol. 16, no. 4, pp. 356-363, 2004.

[15] C. P. Poulsen, G. Vereb, N. Geshi, and A. Schulz, "Inhibition of cytoplasmic streaming by cytochalasin D is superior to paraformaldehyde fixation for measuring FRET between fluorescent protein-tagged Golgi components," Cytometry A, vol. 83, no. 9, pp. 830-838, 2013.

[16] J. Roszik, J. Szöllosi, and G. Vereb, "AccPbFRET: an ImageJ plugin for semi-automatic, fully corrected analysis of acceptor photobleaching FRET images," BMC Bioinformatics, vol. 9, article 346, 2008.

[17] C. A. Schneider, W. S. Rasband, and K. W. Eliceiri, "NIH Image to ImageJ: 25 years of image analysis," Nature Methods, vol. 9, no. 7, pp. 671-675, 2012.

[18] J. Šamaj, M. Braun, F. Baluška, H.-J. Ensikat, Y. Tsumuraya, and D. Volkmann, "Specific localization of arabinogalactanprotein epitopes at the surface of maize root hairs," Plant and Cell Physiology, vol. 40, no. 8, pp. 874-883, 1999.

[19] A. Hassinen, F. M. Pujol, N. Kokkonen et al., "Functional organization of Golgi $\mathrm{N}$ - and $\mathrm{O}$-glycosylation pathways involves $\mathrm{pH}$-dependent complex formation that is impaired in cancer cells," The Journal of Biological Chemistry, vol. 286, no. 44, pp. 38329-38340, 2011.

[20] J. Wang, Y. Ding, J. Wang et al., "EXPO, an exocyst-positive organelle distinct from multivesicular endosomes and autophagosomes, mediates cytosol to cell wall exocytosis in arabidopsis and tobacco cells," Plant Cell, vol. 22, no. 12, pp. 4009-4030, 2010.

[21] Y. Ding, J. Wang, Y.-D. Stierhof, D. G. Robinson, and L. Jiang, "Unconventional protein secretion," Trends in Plant Science, vol. 17, no. 10, pp. 606-615, 2012.
[22] J. I. Heazlewood, P. Durek, J. Hummel et al., "PhosPhAt : a database of phosphorylation sites in Arabidopsis thaliana and a plant-specific phosphorylation site predictor," Nucleic Acids Research, vol. 36, no. 1, pp. D1015-D1021, 2008.

[23] R. C. Randall, G. O. Phillips, and P. A. Williams, "The role of the proteinaceous component on the emulsifying properties of gum Arabic," Food Hydrocolloids, vol. 2, no. 2, pp. 131-140, 1988.

[24] S. Al-Assaf and G. O. Phillips, "Hydrocolloids: structurefunction relationships," Food Science \& Technology, vol. 23, no. 3, pp. 17-20, 2009.

[25] P. A. Williams and G. O. Phillips, “Gum arabic," in Handbook of Hydrocolloids, G. O. Phillips and P. A. Williams, Eds., pp. 252273, Woodhead Publishing, Cambridge, UK, 2009.

[26] A. Dilokpimol and N. Geshi, "Enzymatically modified-Gum arabic using Arabidopsis beta-glucuronosyltransferase has altered emulsion property," in Proceedings of the Annual Plant Biotech Denmark Meeting, p. 7, 2014.

[27] J. L. Czlapinski and C. R. Bertozzi, "Synthetic glycobiology: exploits in the Golgi compartment," Current Opinion in Chemical Biology, vol. 10, no. 6, pp. 645-651, 2006.

[28] B. G. Lane, "Oxalate, germin, and the extracellular matrix of higher plants," The FASEB Journal, vol. 8, no. 3, pp. 294-301, 1994.

[29] L. J. Goodrum, A. Patel, J. F. Leykam, and M. J. Kieliszewski, "Gum arabic glycoprotein contains glycomodules of both extensin and arabinogalactan-glycoproteins," Phytochemistry, vol. 54, no. 1, pp. 99-106, 2000.

[30] E. Shpak, J. F. Leykam, and M. J. Kieliszewski, "Synthetic genes for glycoprotein design and the elucidation of hydroxyprolineO-glycosylation codes," Proceedings of the National Academy of Sciences of the United States of America, vol. 96, no. 26, pp. 14736-14741, 1999.

[31] J. Xu, E. Shpak, T. Gu, M. Moo-Young, and M. Kieliszewski, "Production of recombinant plant gum with tobacco cell culture in bioreactor and gum characterization," Biotechnology and Bioengineering, vol. 90, no. 5, pp. 578-588, 2005.

[32] M. J. Kieliszewski, "Synthetic genes for plant gums and other hydroxyproline-rich glycoproteins," Patent WO 01/78503 A2, 2001. 

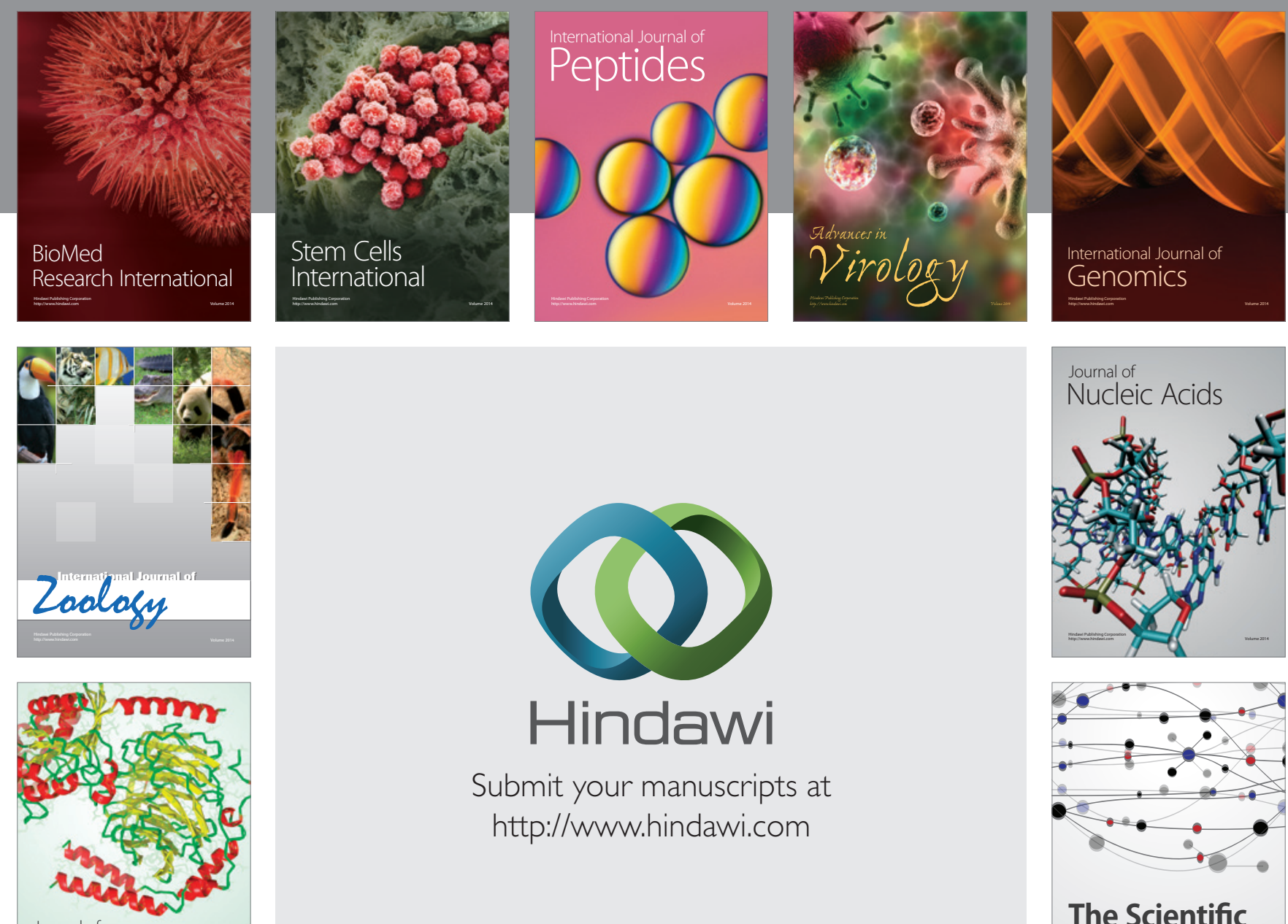

Submit your manuscripts at

http://www.hindawi.com

Journal of
Signal Transduction
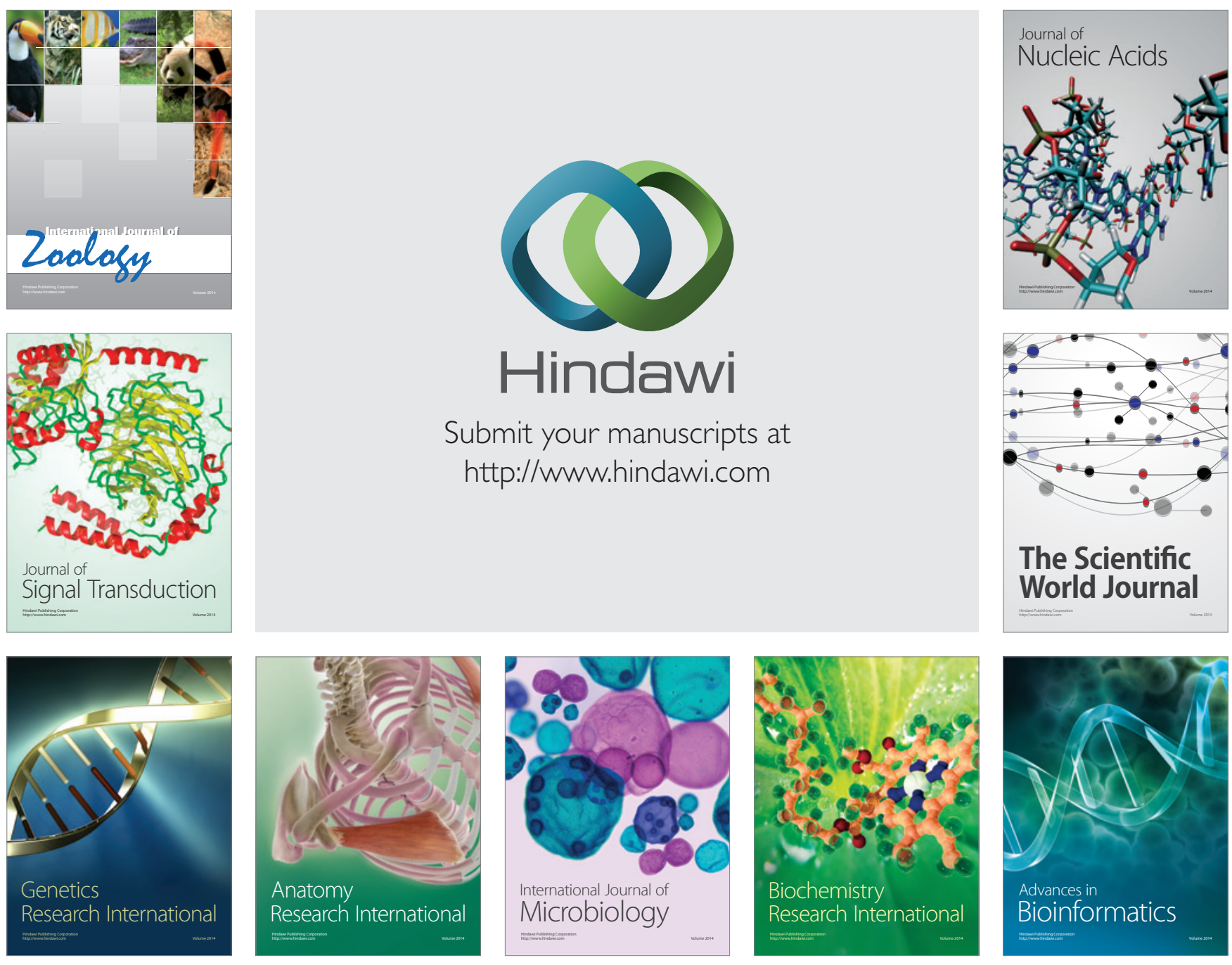

The Scientific World Journal
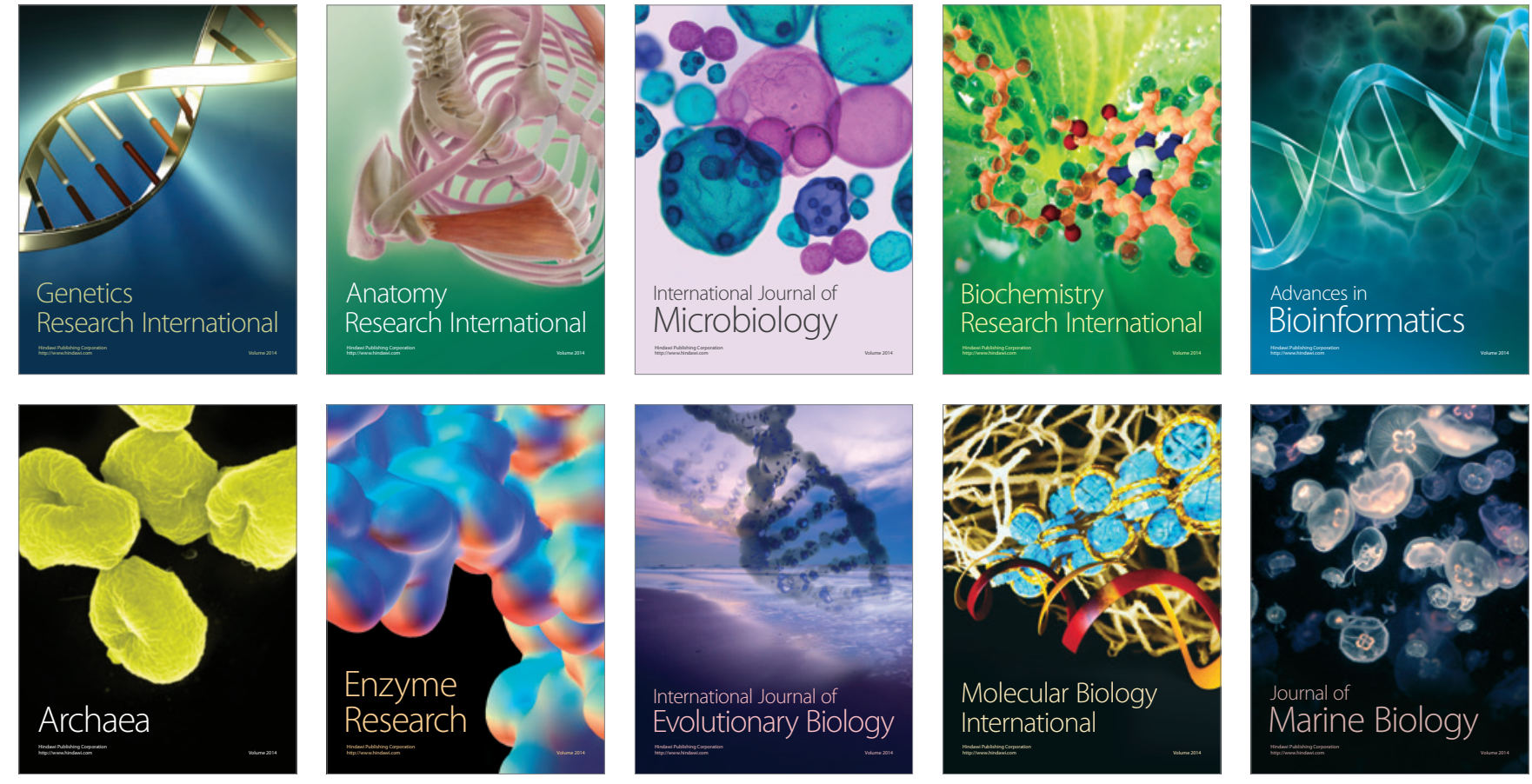